\title{
miR-410 regulates apoptosis by targeting Bak1 in human colorectal cancer cells
}

\author{
CHUNYUAN LIU, AIHONG ZHANG, LEI CHENG and YANG GAO
}

Department of General Surgery, Binzhou Medical University Hospital, Binzhou, Shandong 256603, P.R. China

Received June 29, 2015; Accepted April 26, 2016

DOI: $10.3892 / \mathrm{mmr} .2016 .5271$

\begin{abstract}
MicroRNAs (miRs) are essential in the pathogenesis of colorectal cancer (CRC). Previous studies have demonstrated that miR-410 exerts multiple effects on tumors, however, whether it affects the apoptosis of CRC cells remains to be elucidated. In the present study, to demonstrate the role of miR-410 in CRC, miR-410 expression was detected in CRC tissues and cell lines, and the miR-410 level was manipulated by transfection with an miR-410 or miR-410 inhibitor in CRC cells. Cell growth and apoptosis was tested using an MTT assay, western blot and cytochrome $\mathrm{C}$ assay. Target validation was conducted by luciferase assay. It was found that miR-410 was upregulated in CRC tissues and cell lines. The overexpression of miR-410 resulted in an increase in growth activity and decrease in the extent of apoptosis. By contrast, the inhibition of endogenous miR-410 activated the apoptotic machinery. Western blot analysis and a luciferase activity assay showed that Bak1 was directly targeted by miR-410, and that knockdown of Bak1 attenuated the pro-apoptotic effect of miR-410 inhibition. In addition, it was shown that the expression of Bak1 was downregulated in CRC tumor tissues and was reversely correlated with the expression of miR-410, which provided further support that Bak1 was regulated by miR-410. The results of the present study suggested that miR-410 may function as an oncogenic miR by suppressing the basal level of apoptosis. These findings may assist in understanding the molecular mechanisms of cancer development.
\end{abstract}

\section{Introduction}

Colorectal cancer (CRC) is one of the major causes of cancer-associated mortality worldwide (1). Although the treatment strategy for CRC has improved substantially over the last few decades, targeted therapy for $\mathrm{CRC}$ remains in its infancy (2). In addition, the pathogenesis of $\mathrm{CRC}$ remains to

Correspondence to: Dr Lei Cheng, Department of General Surgery, Binzhou Medical University Hospital, 661 Yellow River Road, Binzhou, Shandong 256603, P.R. China

E-mail: chenglei2081@163.com

Key words: colorectal cancer, apoptosis, microRNA be fully elucidated, and understanding the molecular mechanisms, which drives the development of CRC is essential for identifying novel molecular targets for CRC.

MicroRNAs (miRs) are highly conserved, small non-coding RNA strands, which act as negative regulators of gene expression by incompletely complementary pairing with the 3'-untranslated region (UTR) of target mRNAs, which, in the majority of cases, inhibits the translation process of target genes (3-5). miRs are expressed in a restricted spatial and temporal manner, which confers their ability to specifically regulate different important biological processes in a variety of settings $(6,7)$. Previous investigations have elucidated the pivotal role of miRs in CRC and suggested several novel miR-based therapeutic strategies (8). However, due to the extensive miR-mRNA interaction network in every facet of tumor biology, it is possible that a several additional miRs may be involved in critical processes, including proliferation, apoptosis and trans-differentiation in CRC cells.

B cell lymphoma ( $\mathrm{Bcl})-2$ homologous antagonist/killer (Bak1) is an essential cell death regulator, which initiates mitochondria-mediated apoptosis by interacting with proteins, including Bcl-extra large (xL) (9) and voltage-dependent anion channels (VDACs) (10). Previous studies have shown that $\mathrm{Bcl}-2$ family proteins, including Bcl-2-associated $\mathrm{X}$ protein (Bax), are capable of predicting CRC prognosis, and that Bax agonists efficiently induce apoptosis in tumors (11-14). However, how their expression is intrinsically controlled in $\mathrm{CRC}$ remains to be fully elucidated. In the present study, the miRanda database (www.microrna.org) was used to search for potential microRNAs that target Bak1. It was suggested that Bak1 may be targeted by miR-410; the data in the present study may show a differential expression of miR-410 in CRC tumor tissues and characterize the role of miR-410 in the apoptosis of CRC cells for the first time. These data will help to understand the implication of miR-410 in CRC tumorigenesis and its potential therapeutic value in CRC treatment.

\section{Materials and methods}

Tissue collection. A total of 20 samples of human colorectal adenocarcinoma tumor tissues and their paired adjacent normal tissues were collected from Binzhou Medical University Hospital (Binzhou, China); the tissues were immediately transferred into liquid nitrogen following laparoscopic resection. All patients were diagnosed with adenocarcinoma 
and were aged between 35 and 60 years. Among them, 12 were male and 8 were female. Tumor samples were collected between February and May 2013. The cancer tissues and adjacent tumor tissues were collected, and the negative margin of the adjacent tissue was confirmed by histological examination. All diagnoses were histologically confirmed. Informed consent was obtained from the patients, and the experimental protocol was approved by the institutional Ethics Committee of Binzhou Medical University Hospital, Binzhou, China.

Cell culture and transfection. Cell lines of human colorectal cancer (SW480, HT29 and HCT116) and normal colon epithelial cells (FHC) were obtained from American Type Culture Collection (Vanassas, MA, USA). The cells were cultured in Dulbecco's modified Eagle's medium supplemented with 10\% fetal bovine serum at a temperature of $37^{\circ} \mathrm{C}$ with $5 \% \mathrm{CO}_{2}$. The culture media were replaced every other day. The pan-caspase inhibitor, ZVAD-fmk (Sigma-Aldrich, St. Louis, MO, USA) was dissolved in dimethyl sulfoxide (DMSO) and used at the final concentration of $20 \mu \mathrm{M}$. Small interfering (si)RNA for Bak1, miR-410 mimics, miR-410-inhibitor and their negative control sequences were transfected into cells using Lipofectamine 2000 (Invitrogen; Thermo Fisher Scientific, Inc., Waltham, MA, USA), according to the protocols provided by the manufacturer. Cells at $70-80 \%$ confluence were transfected in the absence of antibiotics. After $24 \mathrm{~h}$ of transfection the medium was replaced. miR-410 and the miR-410-inhibitor were purchased from Guangzhou RiboBio Co., Ltd, (Guangzhou, China), and Bak1-siRNA was purchased from Santa Cruz Biotechnology, Inc. (Santa Cruz, CA, USA).

RNA isolation and reverse transcription-quantitative polymerase chain reaction ( $R T-q P C R)$ analysis. Cells were scraped from the culture plate, and total RNA from the tissues and treated cells was isolated using TRIzol reagent (Invitrogen; Thermo Fisher Scientific, Inc.). The RNA samples were then reverse transcribed using a specific stem-loop primer (Guangzhou RiboBio Co., Ltd.) for miR-410, followed by amplification with SYBR Green master mix on the ABI7000 Real-Time PCR system (Applied Biosystems; Thermo Fisher Scientific, Inc.). The cDNA templates were diluted to $1: 10$ and $5 \mu \mathrm{l}$ per reaction was analyzed. The thermal cycling conditions were as follows: $95^{\circ} \mathrm{C}$ for $10 \mathrm{~min}$, $95^{\circ} \mathrm{C}$ for $15 \mathrm{sec}, 50-60^{\circ} \mathrm{C}$ for $5 \mathrm{sec}$ and $72^{\circ} \mathrm{C}$ for $15 \mathrm{sec}$ for 40 cycles.The Bulge-Loop ${ }^{\mathrm{TM}}$ hsa-miR-410 qRT-PCR primer set was purchased from Guangzhou RiboBio Co., Ltd. U6 small nuclear RNA was used as the internal control to normalize the expression of miR-410. The relative expression of miR-410 was determined using the $2^{-\Delta \Delta \mathrm{Cq}}$ method (15).

Methylthiazolyldiphenyl-tetrazolium bromide (MTT) assay. The SW480 cells were seeded into 96-well plates at $2.5 \times 10^{4} / \mathrm{ml}$, and cell viability was determined by the MTT method using a kit (cat. no. C0009) obtained from Beyotime Institute of Biotechnology (Shanghai, China). The formazan produced by the living cells in the presence of MTT were visualized by DMSO dissolution. The absorbance value at $570 \mathrm{~nm}$ in each group was measured using a microplate reader and used to compare the growth activity of the cells in different groups.
Cytochrome c apoptosis assay. Cytochrome $c$ release was used to determine apoptosis. The present study assessed the release of cytochrome $c$ in the cytosol using an ELISA kit (cat. no. H190; Nanjing Jiancheng Bioengineering Institute, Nanjing, China), according to the manufacturer's protocol. The experiments were performed in triplicate.

Western blot analysis. Protein extraction was performed by homogenizing the cells with radioimmunoprecipitation assay lysis buffer (Beyotime Institute of Biotechnology), followed by mixing with an appropriate quantity ( $1 / 5$ of total sample volume) of SDS-sample buffer (Beyotime Institute of Biotechnology) and heating at $100^{\circ} \mathrm{C}$ for $3 \mathrm{~min}$. Protein was quantified using a BCA Protein Assay kit (cat. no. P0011; Beyotime Institute of Biotechnology). The proteins $(30 \mu \mathrm{g})$ were then subjected to $10 \%$ SDS-PAGE and transferred onto nitrocellulose membranes. The membranes were blocked with $5 \%$ milk for $1 \mathrm{~h}$ at room temperature. The membranes were incubated with the following primary rabbit antibodies at $4^{\circ} \mathrm{C}$ for $16 \mathrm{~h}$ : Anti-Bak1 (1:1,000; cat. no. 12105), anti-Bcl-2 (1:1,000; cat. no. 2876), anti-c-caspase-3 (1:500; cat. no. 9664), anti-VDAC (1:1,000; cat. no. 4866) and anti- $\beta$-actin (1:1,000; cat. no. 4976). The membranes were then incubated with horseradish peroxidase-conjugated rabbit $\mathrm{IgG}$ secondary antibody (1:3,000; cat. no. 7074) at room temperature for $50 \mathrm{~min}$, and the bands were detected using enhanced chemiluminescence methods with a kit (cat. no. P0018; Beyotime Institute of Biotechnology). The membranes were washed with phosphate-buffered saline with $0.5 \%$ Tween 20 three times (10 min each) after primary antibody and secondary antibody incubation. The bands were quantified using ImageJ software (version 1.45; National Institutes of Health, Bethesda, MA, USA). The relative expression levels of the proteins of interest were obtained by normalizing their band densities to that of $\beta$-actin. All antibodies were purchased from Cell Signaling Technology, Inc.

Luciferase assay. A 415bp-fragment of the Bak1 3'-UTR, which contained a putative binding site of miR-410, was subcloned into the 3'-UTR region of the pmirGLO firefly luciferase construct (Promega, Madison, WI, USA). The following primers were used for PCR amplification: Sense 5'-CGAGCT CGTCCTCTCAGTTCTCTCCCT-3' and antisense 5'-GCT CTAGAAGGCTGTGCCCAATAGAGAA-3'. The product was then inserted into the site between $S a c I$ and $X b a I$. The empty vector and the PCR products were digested with SacI and $\mathrm{XbaI}$, and subjected to T4 DNA ligation (Promega) at $16^{\circ} \mathrm{C}$ overnight. The ligation product was then transformed. Single colonies were picked and the construct was confirmed by sequencing. The constructs were then transfected into SW480 cells, accompanied with the internal control vector, pRL-TK, and miR-410 mimics or miR-410-inhibitor (Guangzhou RiboBio Co., Ltd.). At $48 \mathrm{~h}$ post-transfection, the luciferase activity was measured using a Dual-Luciferase Reporter assay kit (cat. no. E1901; Promega).

Statistical analysis. All the data are reported as the mean \pm standard deviation. SPSS version 19.0 (IBM SPSS, Amronk, NY, USA) was used to perform statistical analyses. Differences in the expression levels of miR-410 and Bak1 in 
the paired tissue samples were determined using a paired $t$-test. Comparisons of three or more groups were performed using one way analysis of variance followed by a Newman-Keuls post-hoc test. Correlations between the expression levels of Bak1 and miR-410 were analyzed using Spearman's correlation. Two-tailed $\mathrm{P}<0.05$ was considered to indicate a statistically significant difference.

\section{Results}

miR-410 is upregulated in CRC tumor and cell lines. To evaluate the potential role of miR-410 in CRC, the present study firstly compared the expression levels of miR-410 in CRC tissues and the matched adjacent normal tissues. As shown in Fig. 1A, a significant increase in the expression of miR-410 was observed in the tumor tissues. The expression of miR-410 was also examined in several CRC cell lines, and it was found that miR-410 was expressed at a higher levels in the SW480, HCT-116 and HT-29 CRC cell lines, compared with the FHC normal colon epithelial cell line (Fig. 1B).

miR-410 suppresses apoptosis and promotes growth of CRC cells. To further understand the role of miR-410 in CRC, the present study transfected the SW480 cell line with miR-410 mimics or miR-410 antisense inhibitor to promote the expression of miR-410 or inhibit the endogenous expression of miR-410, respectively. RT-qPCR analysis confirmed the transfection efficiency (Fig. 2A). The transfected SW480 cells were allowed to grow for 4 days consecutively, and the viability of the cells in each group were determined at indicated time points using an MTT assay. As shown in Fig. 2B, the forced expression miR-410 promoted the growth activity of CRC cells, whereas the inhibition of miR-410 decreased the growth activity. Of note, the ZVAD-fmk apoptosis inhibitor markedly attenuated the growth inhibitory effect of the miR-410-inhibitor, suggesting that miR-410 may have altered the growth activity of CRC cells by affecting the apoptotic activity. The results of the western blot analysis of the apoptosis marker, cleaved-caspase-3, are shown in Fig. 2. Consistent with expectations, the overexpression of miR-410 decreased the expression of cleaved-caspase-3, and the inhibition of miR-410 enhanced its expression. The present study also observed alteration in the release of cytochrome $c$ following the gain or loss of function of miR-410, and the results were consistent with the results obtained from the western blot analysis of cleaved-caspase-3 (Fig. 2D).

miR-410 suppresses apoptosis by targeting Bakl in CRC cells. The present study then aimed to determine the mechanism by which miR-410 regulates apoptosis in CRC cells. Bioinformatics analysis predicted a putative binding site of miR-410 in the human Bak1 3'-UTR (Fig. 3A), and the present study assessed whether Bak1 was targeted by miR-410 in the CRC cells. The results of the RT-qPCR analysis showed that the loss or gain of function of miR-410 had no significant affect on the mRNA level of Bak1 (Fig. 3B). The protein level of Bak1 was significantly inhibited when miR-410 was overexpressed, whereas the inhibition of miR-410 had the opposite effect (Fig. 3C). Bcl-2, an anti-apoptotic protein, which is important in the mitochondrial apoptotic pathway, was positively correlated with the expression of miR-410. Previous studies have shown that VDAC is controlled by Bak1 and mediates the release of cytochrome $c$ to facilitate apoptosis $(10,16)$, and the present study observed that the expression of VDAC was in agreement with Bak1, which suggested that the Bak1/VDAC interaction was involved in miR-410-associated apoptotic signaling. Further investigation showed that miR-410 decreased the luciferase activity in the SW480 cells, whereas the inhibition of miR-410 increased the luciferase activity (Fig. 3D). Additionally, as shown in Fig. 3E, the silencing of Bak1 using siRNA significantly attenuated the pro-apoptotic effect of the miR-410 inhibitor on the CRC cells. Taken together, these results showed that Bak1 was a direct target of miR-410 and was required for the apoptosis induced by miR-410 inhibition.

Expression of Bakl is inversely correlated with the expression of miR-410 in CRC. As the Bak1 was confirmed as a direct target of miR-410 in vitro, the present study next investigated whether the expression of Bak1 was also altered in tumor tissues. As shown in Fig. 4A, the expression of Bak1 was found to be downregulated in the CRC tissues, compared with the adjacent tumor tissues. Of note, Spearman's correlation coefficient showed a significant inverse correlation between the expression of Bak1 and miR-410 (Fig. 4B). This result further confirmed that Bak1 was targeted by miR-410 in the clinical setting of CRC.

\section{Discussion}

In the present study, it was demonstrated that miR-410 was crucial in regulating the apoptosis of CRC cells. It was found that miR-410 was upregulated in CRC tissues and cells. Mechanistically, the present study revealed that the pro-apoptotic factor, Bak1, was targeted by miR-410, which in turn decreased the basal level of apoptosis and thus enhanced the growth activity of CRC cells. The results of the present study, which reported the tumorgenicity of miR-410, may not only expand on current understanding of the regulatory mechanism of cell death by non-coding RNAs, but also provided insights into the targeted therapy for CRC.

Accumulating evidence has confirmed that miRs are important regulators either in the early development or in the progress of CRC (8), and previous reports have identified that several miRs are aberrantly expressed $(17,18)$. For example miR-143 and miR-145, which have been confirmed as tumor suppressors, are markedly downregulated in CRC (17,19). By contrast, miR-21 has been identified as an oncogenic miR, which is important in several aspects of CRC, including apoptosis, proliferation and metastasis (20-22). Previous studies have shown that miR-410 acts in a diverse manner to control multiple cellular processes, including angiogenesis, differentiation and proliferation (23-25). However, the roles of miR-410 in regulating cell behaviors remain to be fully elucidated, and conflicting results have been reported with regards to its role in cancer cell growth. Chien et al reported that miR-410 is involved in cell cycle progression by regulating the cyclin-dependent kinase 1-dependent $\mathrm{pRb} / \mathrm{E} 2 \mathrm{~F}$ pathway in breast cancer cells (26). Chen et al reconfirmed the antitumor activity of miR-410 in glioma cells, showing that MET, the 
A

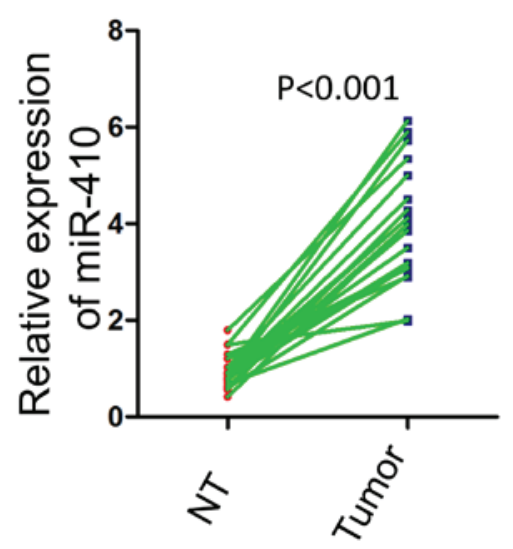

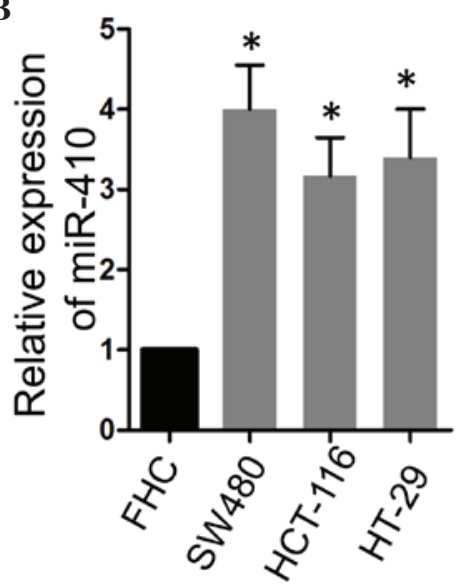

Figure 1. miR-410 is upregulated in CRC tumor tissues and cell lines. (A) Relative expression levels of miR-410 in tumor tissues and matched adjacent normal tissues. (B) Relative expression levels of miR-410 in a normal colon epithelial cell line and CRC cell lines. Data are presented as the mean \pm standard deviation. ${ }^{*} \mathrm{P}<0.05$, vs. FHC. miR, microRNA; CRC, colorectal cancer; NT, normal tissue.

A

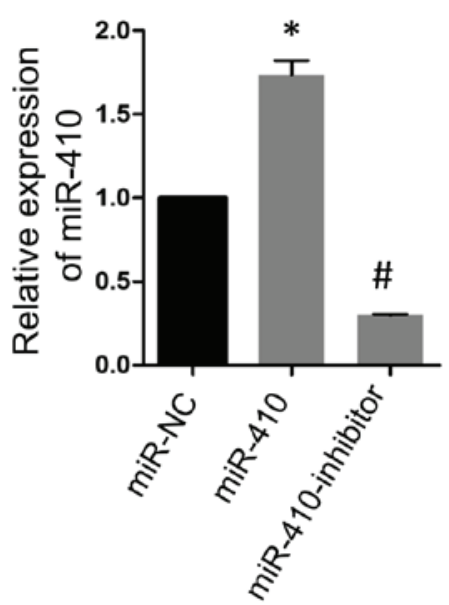

C
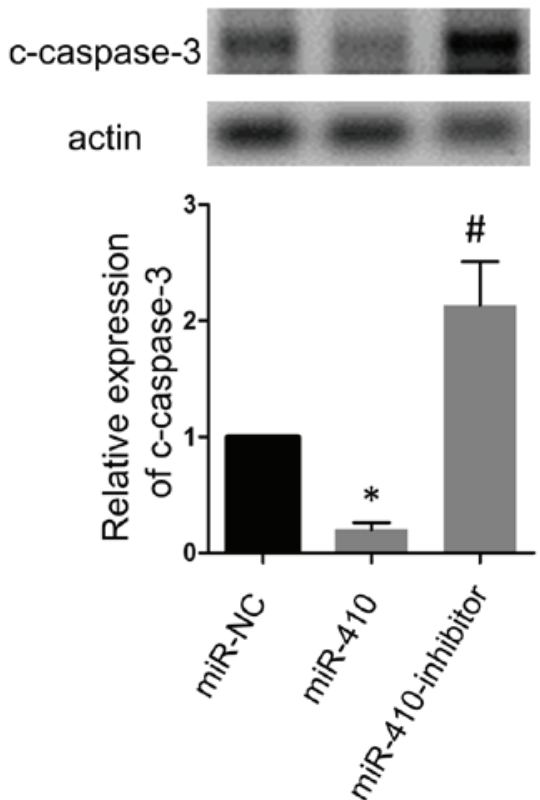

B

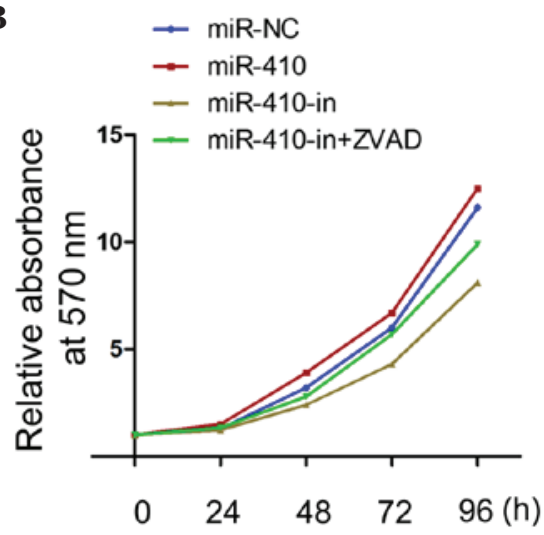

D

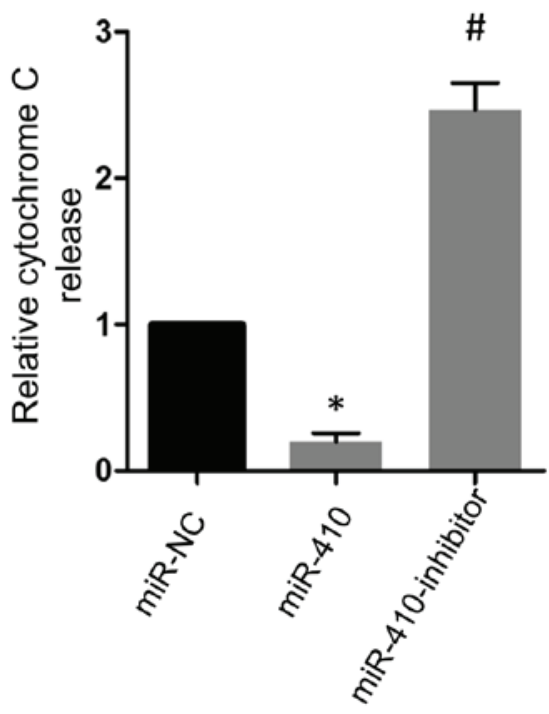

Figure 2. miR-410 suppresses apoptosis and promotes growth of SW480 cells. (A) Relative expression levels of miR-410 following transfection with miR-410 mimic (NC) or miR-410-inhibitor. (B) Effects of miR-410 and the miR-410-inhibitor on cell growth. miR-410 enhanced cell growth activity, whereas miR-410-inhibitor decreased cell growth activity. ZVAD-fmk reduced the growth inhibitory effect of the miR-410-inhibitor. (C) Effects of miR-410 and the miR-410 inhibitor on the apoptosis marker, c-caspase-3, and the (D) release of cytochrome $c$. Data are presented as the mean \pm standard deviation. ${ }^{*} \mathrm{P}<0.05$ and ${ }^{\#} \mathrm{P}<0.05$, vs. miR-NC. miR, microRNA; in, inhibitor; c-caspase-3, cleaved-caspase-3; NC, negative control. 
A

3'-ugUCCGGUA---GACACAAUAUAa-5' hsa-miR-410

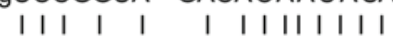

5' -uUAGGACUUGGUUUGUUAUAUc -3' 869-890 of BAK1 3'-UTR
B

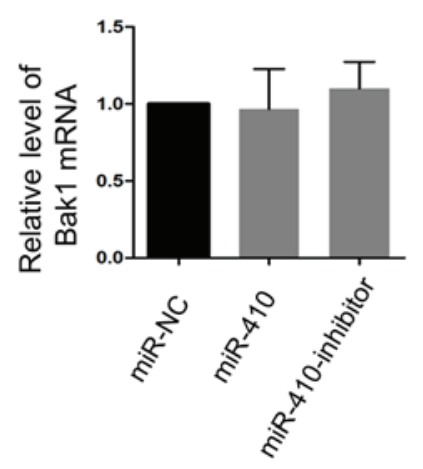

C
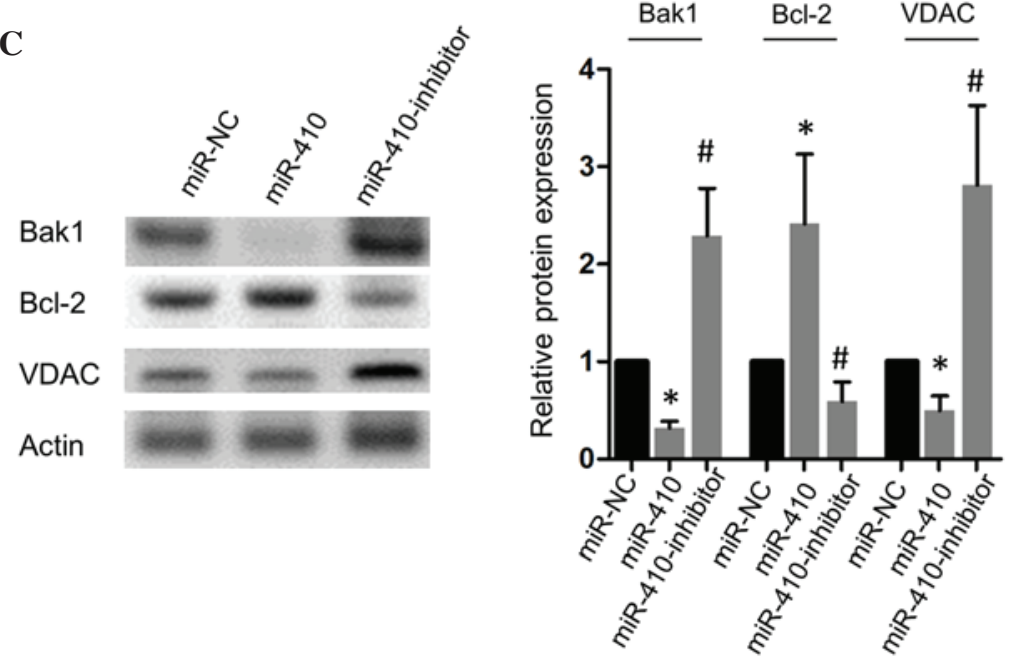

$\mathbf{E}$

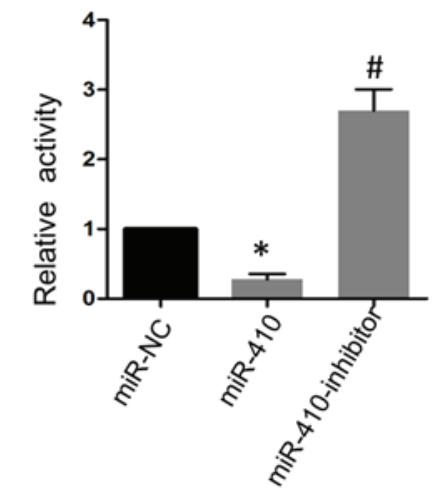

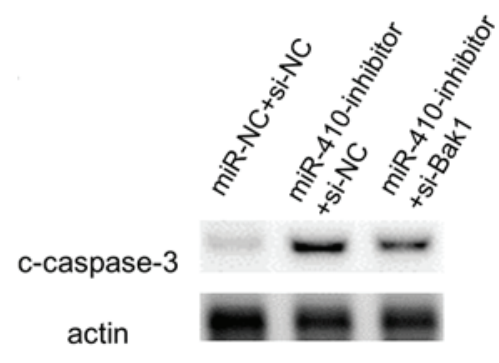
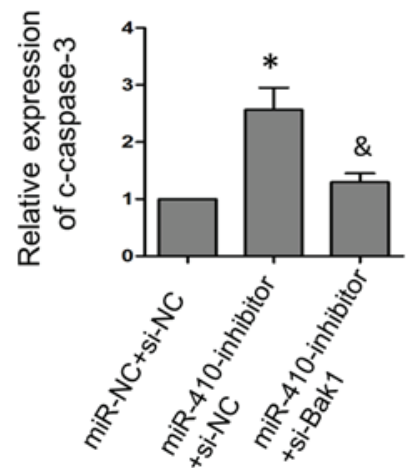

Figure 3. miR-410 suppresses apoptosis by targeting Bak1 in SW480 cells. (A) Schematic showing the putative base-pair binding between miR-410 and the BAK1 3'-UTR. (B) Effects of miR-410 and the miR-410 inhibitor on the mRNA levels of Bak1. (C) Effects of miR-410 and the miR-410 inhibitor on the protein levels of Bak1, Bcl-2 and VDAC. (D) Effects of miR-410 and the miR-410 inhibitor on the luciferase activity of the pmirGLO-BAK1-3'-UTR in SW480 cells. (E) Silencing Bak1 attenuated the caspase-3 cleavage induced by the miR-410-inhibitor. ${ }^{*} \mathrm{P}<0.05$ and ${ }^{\text {}} \mathrm{P}<0.05$, vs. miR-NC or miR-NC+si-NC. ${ }^{\&} \mathrm{P}<0.05$, vs. miR-410-inhibitor+si-NC. miR, microRNA; Bak1, B cell lymphoma-2-agonist/killer 1; VADC, voltage-dependent anion channel; c-caspase-3, cleaved-caspase-3; NC, negative control; UTR, untranslated region; si-, small interfering-RNA.

A

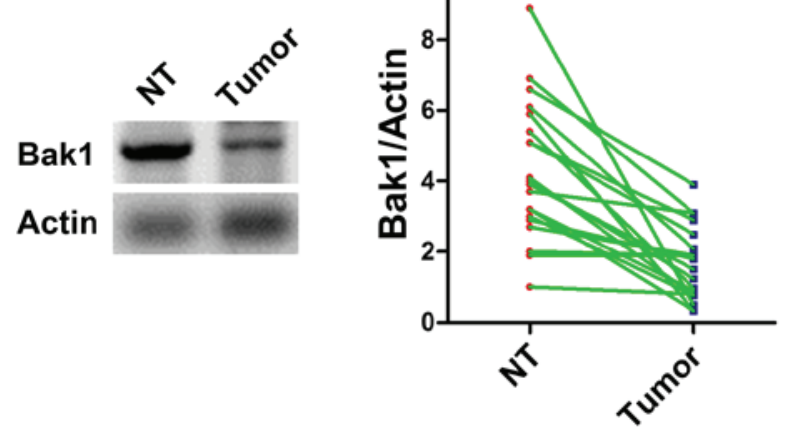

B

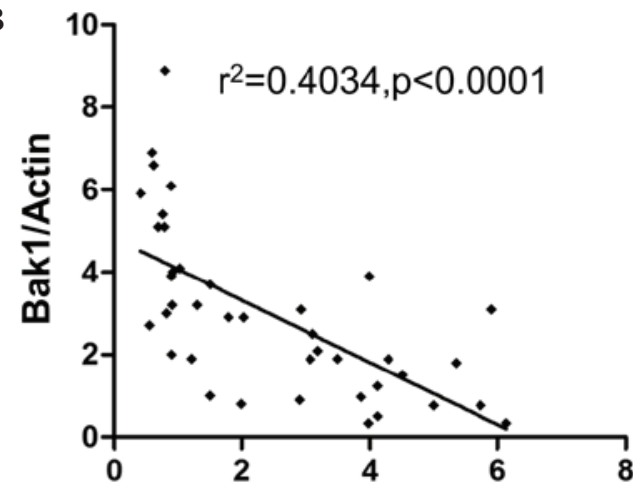

Relative miR-410 expression

Figure 4. Expression of Bak1 is inversely correlated with the expression of miR-410 in patients with colorectal cancer. (A) Relative expression levels of Bak1 in tumor tissues and matched adjacent normal tissues. (B) Correlation analysis of the expression of Bak1 and miR-410. miR, microRNA; Bak1, B cell lymphoma-2-agonist/killer 1; NT, normal tissue. 
receptor for hepatocyte growth factor receptor, is targeted by miR-410 (27). Notably, a study by Wang et al demonstrated the increased expression and oncogenic role of miR-410 in hepatic and colorectal tumors; they suggested that miR-410 binds to the 3'-UTR of four and a half LIM domains 1, which promoted its methylation and thus inhibited its transcription (28). The divergent conclusions of these studies suggest that the role of miR-410 in cancer may be temporally- or spatially-specific. Despite reports of its role in CRC, whether miR-410 functions to regulate the apoptotic machinery remain to be elucidated. In agreement with Wang et al (28), the present study demonstrated that miR-410 enhanced the growth activity of CRC cells. However, these results are the first, to the best of our knowledge, to suggest that miR-410 may be a component of the apoptotic regulatory network, as the apoptosis inhibitor, ZVAD-fmk, efficiently inhibited the growth inhibitory activity of the miR-410 inhibitor, and altered the activation of caspase- 3 by miR-410 gain or loss of function.

miRs are important negative regulators of gene expression. Using bioinformatics analysis, the present study found a segment of complementary sequence of miR- 410 within the 3'-UTR of the Bak1-encoding gene. Therefore, it was suggested that Bak1 was a putative functional target in this cellular model. Previous studies have shown that Bak1 is a critical regulator of mitochondria-mediated apoptosis $(9,10)$. Bak1 and Bax, two pro-apoptotic proteins, form a complex upon apoptotic stimuli, which mediates the release of cytochrome $c$ (10). It has also been shown that several Bcl-2 family proteins, including Bak1 and Bax, act downstream of the transcriptional factor, p53 (9). As p53 mutations are often observed in CRC (29-31), it is possible that the function of Bak1 may have important implications in CRC. Although several studies have preliminarily characterized the role of Bak1 in vitro and in vivo (32-34), how the expression of Bak1 is regulated under the circumstance remains to be fully elucidated. miRs constitute important regulators in apoptosis, and a previous study showed that Bak1 is targeted by miR-125b and mediates its anti-apoptotic effect in neural crest cells (35). Consistent with the previous reports, the present study provided compelling evidence that Bak1 is an important regulator of apoptosis, which is under the control of miR-410. miRs can have multiple targets and, in the present study, it was shown that miR-410 not only altered the expression of Bak1, but also affected the expression of $\mathrm{Bcl}-2$ and VDAC. Whether these changes were the result of alterations in the expression of Bak1 or the functional readout by other possible uncharacterized target genes remains to be elucidated. However, the overall anti-apoptotic effect of miR-410 suggests its potential application in cancer treatment.

Of note, the present study demonstrated that miR-410 is upregulated in CRC tissues, compared with normal adjacent tissues, and was well correlated with the expression of Bak1. However, how miR-410 is constantly regulated under oncogenic stimuli remains to be elucidated. Previous studies have demonstrated that the expression of miRs can be regulated at the transcriptional level. For example, miR-34a is transactivated by p53, which exerts multiple antitumor effects in CRC (36-38), and the expression of miR-21 expression is induced by AP-1 during tumorgenesis (39). As miR-410 is aberrantly regulated in $\mathrm{CRC}$, identifying the nodal points, which regulate its expression may be a suitable topic for future investigation.

In conclusion, the present study identified miR-410 as an miR with tumorigenic potential, and provided compelling evidence that miR-410 is integrated into the apoptotic program of CRC. It was shown that the upregulated expression of miR-410 may contribute to decreased levels of basal apoptosis, which may be the possible mechanism driving the development of CRC. These results may assist in the development of targeted molecular therapy for CRC.

\section{References}

1. Jemal A, Bray F, Center MM, Ferlay J, Ward E and Forman D: Global cancer statistics. CA Cancer J Clin 61: 69-90, 2011.

2. Hagan S, Orr MC and Doyle B: Targeted therapies in colorectal cancer-an integrative view by PPPM. EPMA J 4: 3, 2013.

3. Bartel DP: MicroRNAs: Genomics, biogenesis, mechanism, and function. Cell 116: 281-297, 2004.

4. Calin GA and Croce CM: MicroRNA signatures in human cancers. Nat Rev Cancer 6: 857-866, 2006.

5. Bartel DP: MicroRNAs: Target recognition and regulatory functions. Cell 136: 215-233, 2009.

6. Ambros V: The functions of animal microRNAs. Nature 431: 350-355, 2004.

7. Kloosterman WP and Plasterk RH: The diverse functions of microRNAs in animal development and disease. Dev Cell 11: 441-450, 2006.

8. Schetter AJ, Okayama H and Harris CC: The role of microRNAs in colorectal cancer. Cancer J 18: 244-252, 2012.

9. Nieminen AI, Eskelinen VM, Haikala HM, Tervonen TA, Yan Y, Partanen JI and Klefström J: Myc-induced AMPK-phospho p53 pathway activates Bak to sensitize mitochondrial apoptosis. Proc Natl Acad Sci USA 110: E1839-E1848, 2013.

10. Shimizu S, Narita M and Tsujimoto Y: Bcl-2 family proteins regulate the release of apoptogenic cytochrome $c$ by the mitochondrial channel VDAC. Nature 399: 483-487, 1999.

11. Pryczynicz A, Gryko M, Niewiarowska K, Cepowicz D, Ustymowicz M, Kemona A and Guzińska-Ustymowicz K: Bax protein may influence the invasion of colorectal cancer. World J Gastroenterol 20: 1305-1310, 2014.

12. García-Flórez LJ, Gómez-Álvarez G, Frunza AM, Barneo-Serra L, Martínez-Alonso C and Fresno-Forcelledo MF: Predictive markers of response to neoadjuvant therapy in rectal cancer. J Surg Res 194: 120-126, 2015.

13. Xin M, Li R, Xie M, Park D, Owonikoko TK, Sica GL, Corsino PE, Zhou J, Ding C, White MA, et al: Small-molecule Bax agonists for cancer therapy. Nat Commun 5: 4935, 2014.

14. Ogura E, Senzaki H, Yamamoto D, Yoshida R, Takada H, Hioki K and Tsubura A: Prognostic significance of Bcl-2, Bcl-xL/S, Bax and Bak expressions in colorectal carcinomas. Oncol Rep 6: 365-369, 1999.

15. Livak KJ and Schmittgen TD: Analysis of relative gene expression data using real-time quantitative PCR and the $2^{-\Delta \Delta \mathrm{Ct}}$ method. Methods 25: 402-408, 2001.

16. Naghdi S, Varnai P and Hajnoczky G: Motifs of VDAC2 required for mitochondrial Bak import and tBid-induced apoptosis. Proc Natl Acad Sci USA 112: E5590-E5599, 2015.

17. Michael MZ, O' Connor SM, van Holst Pellekaan NG, Young GP and James RJ: Reduced accumulation of specific microRNAs in colorectal neoplasia. Mol Cancer Res 1: 882-891, 2003.

18. Luo X, Burwinkel B, Tao S and Brenner H: MicroRNA signatures: Novel biomarker for colorectal cancer? Cancer Epidemiol Biomarkers Prev 20: 1272-1286, 2011.

19. Akao Y,Nakagawa Y,Hirata I,Iio A,Itoh T, Kojima K, Nakashima R, Kitade Y and Naoe T: Role of anti-oncomirs miR-143 and -145 in human colorectal tumors. Cancer Gene Ther 17: 398-408, 2010.

20. Asangani IA, Rasheed SA, Nikolova DA, Leupold JH, Colburn NH, Post S and Allgayer H: MicroRNA-21 (miR-21) post-transcriptionally downregulates tumor suppressor Pdcd4 and stimulates invasion, intravasation and metastasis in colorectal cancer. Oncogene 27: 2128-2136, 2008.

21. Peacock O, Lee AC, Cameron F, Tarbox R, Vafadar-Isfahani N, Tufarelli C and Lund JN: Inflammation and MiR-21 pathways functionally interact to downregulate PDCD4 in colorectal cancer. PloS One 9: e110267, 2014. 
22. Lin PL, Wu DW, Huang CC, He TY, Chou MC, Sheu GT and Lee H: MicroRNA-21 promotes tumour malignancy via increased nuclear translocation of $\beta$-catenin and predicts poor outcome in APC-mutated but not in APC-wild-type colorectal cancer. Carcinogenesis 35: 2175-2182, 2014.

23. Shen J, Niu W, Zhou M, Zhang H, Ma J, Wang L and Zhang H: MicroRNA-410 suppresses migration and invasion by targeting MDM2 in gastric cancer. PloS One 9: e104510, 2014.

24. Chen N, Wang J, Hu Y, Cui B, Li W, Xu G, Liu L and Liu S: MicroRNA-410 reduces the expression of vascular endothelial growth factor and inhibits oxygen-induced retinal neovascularization. PloS One 9: e95665, 2014.

25. Choi SW, Kim JJ, Seo MS, Park SB, Kang TW, Lee JY, Lee BC, Kang I, Shin TH, Kim HS, et al: miR-410 inhibition induces RPE differentiation of amniotic epithelial stem cells via overexpression of OTX2 and RPE65. Stem Cell Rev 11: 376-386, 2015.

26. Chien WW, Domenech C, Catallo R, Kaddar T, Magaud JP Salles $\mathrm{G}$ and Ffrench $\mathrm{M}$ : Cyclin-dependent kinase 1 expression is inhibited by p16(INK4a) at the post-transcriptional level through the microRNA pathway. Oncogene 30: 1880-1891, 2011

27. Chen L, Zhang J, Feng Y, Li R, Sun X, Du W, Piao X, Wang H, Yang D, Sun Y, et al: MiR-410 regulates MET to influence the proliferation and invasion of glioma. Int J Biochem Cell Biol 44: $1711-1717,2012$

28. Wang Y, Fu J, Jiang M, Zhang X, Cheng L, Xu X, Fan Z, Zhang J, Ye Q and Song H: MiR-410 is overexpressed in liver and colorectal tumors and enhances tumor cell growth by silencing FHL1 via a direct/indirect mechanism. PloS One 9: e108708, 2014.

29. Liu Y and Bodmer WF: Analysis of P53 mutations and their expression in 56 colorectal cancer cell lines. Proc Natl Acad Sci USA 103: 976-981, 2006.

30. Oden-Gangloff A, Di Fiore F, Bibeau F, Lamy A, Bougeard G, Charbonnier F, Blanchard F, Tougeron D, Ychou M, Boissière F, et al: TP53 mutations predict disease control in metastatic colorectal cancer treated with cetuximab-based chemotherapy. Br J Cancer 100: 1330-1335, 2009.
31. Luo HY and $\mathrm{Xu} \mathrm{RH}$ : Predictive and prognostic biomarkers with therapeutic targets in advanced colorectal cancer. World J Gastroenterol 20: 3858-3874, 2014.

32. Baltaziak M, Koda M, Wincewicz A, Sulkowska M, Kanczuga-Koda L and Sulkowski S: Relationships of P53 and Bak with EPO and EPOR in human colorectal cancer. Anticancer Res 29: 4151-4156, 2009.

33. Kondo S, Shinomura Y, Miyazaki Y, Kiyohara T, Tsutsui S, Kitamura S, Nagasawa Y, Nakahara M, Kanayama S and Matsuzawa Y: Mutations of the bak gene in human gastric and colorectal cancers. Cancer research 60: 4328-4330, 2000.

34. Krajewska M, Moss SF, Krajewski S, Song K, Holt PR and Reed JC: Elevated expression of Bcl-X and reduced Bak in primary colorectal adenocarcinomas. Cancer Res 56: 2422-2427, 1996.

35. Chen X, Liu J, Feng WK, Wu X and Chen SY: MiR-125b protects against ethanol-induced apoptosis in neural crest cells and mouse embryos by targeting Bak 1 and PUMA. Exp Neurol 271: 104-111, 2015.

36. Chang TC, Wentzel EA, Kent OA, Ramachandran K, Mullendore M, Lee KH, Feldmann G, Yamakuchi M, Ferlito M, Lowenstein CJ, et al: Transactivation of miR-34a by p53 broadly influences gene expression and promotes apoptosis. Mol Cell 26: 745-752, 2007.

37. Gao J, Li N, Dong Y, Li S, Xu L, Li X, Li Y, Li Z, Ng SS, Sung JJ, et al: $\mathrm{miR}-34 \mathrm{a}-5 \mathrm{p}$ suppresses colorectal cancer metastasis and predicts recurrence in patients with stage II/III colorectal cancer. Oncogene 34: 4142-4152, 2015.

38. Rokavec M, Öner MG, Li H, Jackstadt R, Jiang L, Lodygin D, Kaller M, Horst D, Ziegler PK, Schwitalla S, et al: IL-6R/STAT3/miR-34a feedback loop promotes EMT-mediated colorectal cancer invasion and metastasis. J Clin Invest 124: $1853-1867,2014$

39. Talotta F, Cimmino A, Matarazzo MR, Casalino L, De Vita G, D'Esposito M, Di Lauro R and Verde P: An autoregulatory loop mediated by miR-21 and PDCD4 controls the AP-1 activity in RAS transformation. Oncogene 28: 73-84, 2009. 